\title{
Void-mediated coherency-strain relaxation and impediment of cubic-to-hexagonal transformation in epitaxial metastable metal/semiconductor TiN/Al ${ }_{0.72} \mathrm{Sc}_{0.28} \mathrm{~N}$ multilayers
}

\author{
Magnus Garbrecht, ${ }^{1, *}$ Lars Hultman, ${ }^{1}$ Mohammed H. Fawey, ${ }^{2,3}$ Timothy D. Sands, ${ }^{4}$ and Bivas Saha ${ }^{5}$ \\ ${ }^{1}$ Thin Film Physics Division, Department of Physics, Chemistry, and Biology (IFM), Linköping University, S-58183 Linköping, Sweden \\ ${ }^{2}$ Karlsruhe Institute of Technology (KIT), Institute of Nanotechnology (INT), Hermann-von-Helmholtz-Platz 1, \\ D-76344 Eggenstein-Leopoldshafen, Germany \\ ${ }^{3}$ Joint Research Laboratory Nanomaterials (KIT and TUD) at Technische Universität Darmstadt (TUD), \\ Jovanka-Bontschits-Strasse 2, D-64287 Darmstadt, Germany \\ ${ }^{4}$ Bradley Department of Electrical and Computer Engineering and Department of Materials Science and Engineering, \\ Virginia Tech, Blacksburg, Virginia 24061, USA \\ ${ }^{5}$ Department of Materials Science and Engineering, University of California, Berkeley, California 94720, USA
}

(Received 16 May 2017; published 17 August 2017)

\begin{abstract}
Bulk metastable phases can be stabilized during thin-film growth by employing substrates with similar crystal structure and lattice parameter, albeit over a thickness range limited by coherency-strain relaxation. Expanding that strategy, growth of superlattices comprising one stable and another metastable compound with similar crystal structure and lattice parameters are known to yield epitaxial stabilization over a few nanometers of thickness. In this work, the high-pressure rocksalt (B1) phase of $\mathrm{Al}_{0.72} \mathrm{Sc}_{0.28} \mathrm{~N}$ was stabilized epitaxially in a multilayer with TiN with thicknesses of up to $26 \mathrm{~nm}$. In order to investigate the microstructural changes leading to the phase transformation of the metastable B1 phase to its wurtzite allomorph, we demonstrate a design based on a multilayer architecture with systematically varying thicknesses of the metastable compound within a constant-thickness lattice of stable metallic TiN with the cubic rocksalt structure. The multilayer films show an increasing hardness and elastic modulus for decreasing period thickness, in correspondence with both coherency-strain and Koehler hardening. The phase transition is accompanied by an increase of lattice strain with increasing multilayer periods, and resulting ultimately in coherency-strain relaxation upon phase transformation. Further, we show that the phase transformation is mediated by voids decorating the $\{130\}$ planes that separate regions of different growth rates and act as additional growth fronts for wurtzite growth during the phase transformation. The TiN/(Al,Sc)N interfaces themselves remain atomically sharp and smooth until the interface structure roughens along with the epitaxial rocksalt to wurtzite transition of $(\mathrm{Al}, \mathrm{Sc}) \mathrm{N}$. These results show the strong influence of the voids on controlling the target thickness of epitaxially stabilized thin-film growth to the range relevant for applications, such as coatings, plasmonic materials, and electronic device technology, where the mechanical integrity of the material is critical.
\end{abstract}

DOI: 10.1103/PhysRevMaterials.1.033402

\section{INTRODUCTION}

Unlike most transition-metal mononitrides that crystallize in the cubic rocksalt (B1) phase at equilibrium, commonly known III-V nitride semiconductors such as AlN, GaN, and InN exhibit the hexagonal wurtzite phase under ambient conditions but can be stabilized in their cubic phases under high hydrostatic pressures [1-5]. This form of stabilization is interesting because the B1 phase of the semiconductors possesses different electrical, optical, and mechanical characteristics as compared to the wurtzite phase [6,7]. A well-known approach to stabilize a metastable B1 phase during thin-film growth without applying high pressures is by choice of a substrate with a similar lattice parameter and structure to the desired one, termed epitaxial stabilization, which was realized for,

\footnotetext{
* Author to whom correspondence should be addressed: magnus.garbrecht@liu.se

Published by the American Physical Society under the terms of the Creative Commons Attribution 4.0 International license. Further distribution of this work must maintain attribution to the author(s) and the published article's title, journal citation, and DOI.
}

e.g., GaN inside GaN/TiN and AlN inside AlN/TiN multilayers [8-10]. Furthermore, it was recently shown that alloying AlN with semiconducting $\mathrm{B} 1 \mathrm{ScN}$ yields stable $\mathrm{B} 1 \mathrm{Al}_{x} \mathrm{Sc}_{1-x} \mathrm{~N}$ thin films $[11,12]$, which moreover can be lattice matched with metallic TiN to yield metal/semiconductor superlattices [13-15].

The aim of this work is to study the microstructural changes accompanying or preceding the rocksalt-to-wurtzite phase transformation, and to characterize the coherency-strain relaxation that is expected to happen just before the transformation occurs beyond the critical thickness value. For that purpose, the $\mathrm{B} 1$ phase of $(\mathrm{Al}, \mathrm{Sc}) \mathrm{N}$ was stabilized epitaxially within a multilayer with TiN. In order to evoke the rocksalt-to-wurtzite phase transition, we designed the multilayer architecture with a systematic increase in thicknesses of the metastable $(\mathrm{Al}, \mathrm{Sc}) \mathrm{N}$ compound within a constant-thickness lattice of stable metallic TiN with cubic B1 structure, grown on a $\mathrm{MgO}(001)$ substrate.

Members of the class of metal/semiconductor superlattice materials are of considerable technological interest due to their unique properties and consequently high variety of potential application [16,17]. We have recently demonstrated that transition-metal nitrides such as TiN, HfN, and ZrN can be grown as a constituent of single-crystal thin-film epitaxial metal/semiconductor superlattices on $\mathrm{MgO}(001)$ substrates 
with low defect densities, exhibiting high melting points and mechanical hardness for applications as hard and thermally stable coatings and high-temperature thermoelectric materials [18-22]. In addition to its high chemical and thermal stability, a metal/dielectric $\mathrm{TiN} /(\mathrm{Al}, \mathrm{Sc}) \mathrm{N}$ superlattice architecture has been proven to be a promising hyperbolic metamaterial in the visible spectral range with a large enhancement of its densities of photon states, as is useful in quantum electronic and optoelectronic applications [15,23]. Moreover, TiN/(Al,Sc)N exhibits continuously tunable plasmonic resonances in the visible light spectrum, which is of high importance for energy-related applications [24]. Hence, controlled thin-film growth of stable B1 superlattices is crucial for a wide range of applications, and a better understanding of the phase transition and limiting critical thickness to stabilize metastable phases of the semiconducting constituent is desirable.

\section{EXPERIMENTAL DETAILS}

Epitaxial TiN/(Al,Sc)N metal/semiconductor multilayers were deposited on [001]-oriented $\mathrm{MgO}$ substrates at $750{ }^{\circ} \mathrm{C}$ in a $10 \mathrm{mTorr} \mathrm{Ar} / \mathrm{N}_{2}$ ( $4 \mathrm{sccm} \mathrm{Ar:} 6 \mathrm{sccm} \mathrm{N}$ ) ambient via reactive DC-magnetron sputtering (PVD Products, Inc.) from scandium $(99.998 \%$ purity), aluminum $(99.99 \%)$, and titanium $(99.99 \%)$ targets in a top-down confocal arrangement. The growth chamber was equipped with four targets, three DC power supplies, and the base pressure was $<7 \times 10^{-6} \mathrm{~Pa}$ $\left(5 \times 10^{-8}\right.$ Torr $)$ prior to deposition. The magnetron sputtering was performed in constant power mode (with $\mathrm{Al}=$ $200 \mathrm{~W}, \mathrm{Sc}=180 \mathrm{~W}, \mathrm{Ti}=200 \mathrm{~W}$ ) with a target to substrate distance of $9 \mathrm{~cm}$ and a substrate rotation speed of $5 \mathrm{rpm}$. While the thickness of all TiN layers was aimed to a constant $20 \mathrm{~nm}$, the lowermost $(\mathrm{Al}, \mathrm{Sc}) \mathrm{N}$ layer thickness was $2 \mathrm{~nm}$ and increased in steps of $2 \mathrm{~nm}$ with each additional layer, yielding a layered stack with these features: $\mathrm{MgO}$ substrate/ $20 \mathrm{~nm}$ $\mathrm{TiN} / 2 \mathrm{~nm}(\mathrm{Al}, \mathrm{Sc}) \mathrm{N} / 20 \mathrm{~nm} \mathrm{TiN} / 4 \mathrm{~nm}(\mathrm{Al}, \mathrm{Sc}) \mathrm{N} / 20 \mathrm{~nm} \mathrm{TiN} / 6$ $\mathrm{nm}(\mathrm{Al}, \mathrm{Sc}) \mathrm{N} / 20 \mathrm{~nm} \mathrm{TiN} / 8 \mathrm{~nm}(\mathrm{Al}, \mathrm{Sc}) \mathrm{N}$, and so forth. In order to assure epitaxial growth of the multilayers, lattice match to rocksalt TiN with nominal lattice parameter of $a=0.424 \mathrm{~nm}$ (confirmed by XRD) was achieved by yielding a semiconductor layer composition of $\mathrm{Al}_{0.72} \mathrm{Sc}_{0.28} \mathrm{~N}$, which was confirmed by scanning transmission electron microscopy (STEM)-based energy-dispersive $\mathrm{x}$-ray spectroscopy (EDS) measurements.

Conventional TEM bright- and dark-field (BF/DF) imaging and diffraction was performed with an FEI Tecnai G2 microscope operated at $200 \mathrm{kV}$. All high-resolution TEM, scanning (S)TEM, and EDS mapping experiments where conducted with Linköping's image- and probe-corrected and monochromated FEI Titan 3 60-300 microscope equipped with a high-brightness XFEG source and Super-X EDS detector system for ultrahigh count rates, operated at $300 \mathrm{kV}$. Spatial resolutions in both image- and probe-corrected mode were around $0.7 \AA$, and EDS maps feature count rates above 500000 counts, giving an uncertainty of about $3 \%$ in film composition determination employing the $k$-factor method.

Mapping tensile and compressive strain from atomically resolved images was done employing geometric phase analysis (GPA) using a Digital Micrograph script written by Koch [25]. Atomically resolved STEM micrographs of the same magnification were recorded of each individual TiN layer up to the 14th multilayer period. In order to account for scanning distortion, numerous $(>50)$ ultrafast scanned micrographs with dwell times of $5 \mu$ s per pixel were acquired and averaged by summation and background subtraction. The strain maps show the $x$ and $y$ components of the strain tensor $\left(\varepsilon_{x x}, \varepsilon_{y y}\right)$, respectively. The color scale numbers for the strain maps are given in percent of strain divided by 100. Positive numbers mean tensile, and negative numbers compressive strain.

A cross-section TEM lamella was prepared by focused ion beam (FIB) employing an FEI Strata 400S system equipped with an OmniProbe 200 micromanipulator for in situ lift-out and transfer to a copper grid. The preparation was initially performed at $30 \mathrm{kV}$ with an ion beam current of $21 \mathrm{nA}$. The lamella was thinned to about $100 \mathrm{~nm}$ thickness using ion beam currents from $0.44 \mathrm{nA}$ down to $26 \mathrm{pA}$, and finishing at $5 \mathrm{kV}$ with $15 \mathrm{pA}$ ion beam current. To improve the surface quality and to reduce the thickness further, the lamella was polished with a focused argon ion beam using a NanoMill system (Model 1040, Fischione Instruments). Ion energies from $900 \mathrm{eV}$ down to $600 \mathrm{eV}$ were used for $50 \mathrm{~min}$ in total to remove both the implanted $\mathrm{Ga}$ ions and the amorphous layers stemming from the FIB preparation.

The mechanical properties of the superlattices and individual reference thin films were measured by nanoindentation with a Hysitron Triboindenter 950 equipped with a Berkovich probe with a radius of approximately $150 \mathrm{~nm}$. Forty-nine indents, arrayed in a square, were made in each sample using a load-controlled, partial-unloading method with a peak load of $2000 \mu \mathrm{N}$. Indents were spaced $20 \mu \mathrm{m}$ apart so that the plastic zone of a previous indent did not influence the subsequent indent. The contact radius of each indent at maximum load was less than half of the total film thickness; hence the mechanical properties of the substrate should not impact the measured film properties.

\section{RESULTS AND DISCUSSION}

Figure 1(a) shows a low-magnification overview highangle annular dark-field (HAADF) STEM micrograph of the entire multilayer stack. Due to the $Z$-contrast sensitivity of the technique, the TiN layers appear bright with respect to the lower atomic number $\mathrm{Al}_{0.72} \mathrm{Sc}_{0.28} \mathrm{~N}$ layers. The boundary between the well-defined epitaxial multilayer in the rocksalt phase and the polycrystalline wurtzite regions beyond the critical thickness is sharply defined by a zigzag line. As can be seen from the high-resolution STEM micrograph in the inset, the cubic rocksalt structure is epitaxially stabilized in the $(\mathrm{Al}, \mathrm{Sc}) \mathrm{N}$ layers. Moreover, it is shown that the individual layer thicknesses that were yielded during growth are well reproduced, and the interfaces are smooth and atomically flat. The inset electron diffraction pattern (EDP) of the entire stack shows cubic spots as expected for the rocksalt phase, as well as diffuse but well-defined rings corresponding to polycrystalline regions. Defects can be found running diagonally from the substrate/film interface upwards, running at angles of $\sim 71.5^{\circ}$.

As is confirmed by DF imaging employing the 200 reflection and selected area EDPs in Fig. 1(b), the entire region beyond the sharp zigzag boundary line is polycrystalline, and the microstructure together with the FFT is shown in Fig. 1(c). From the EDP of Fig. 2(b) a lattice 

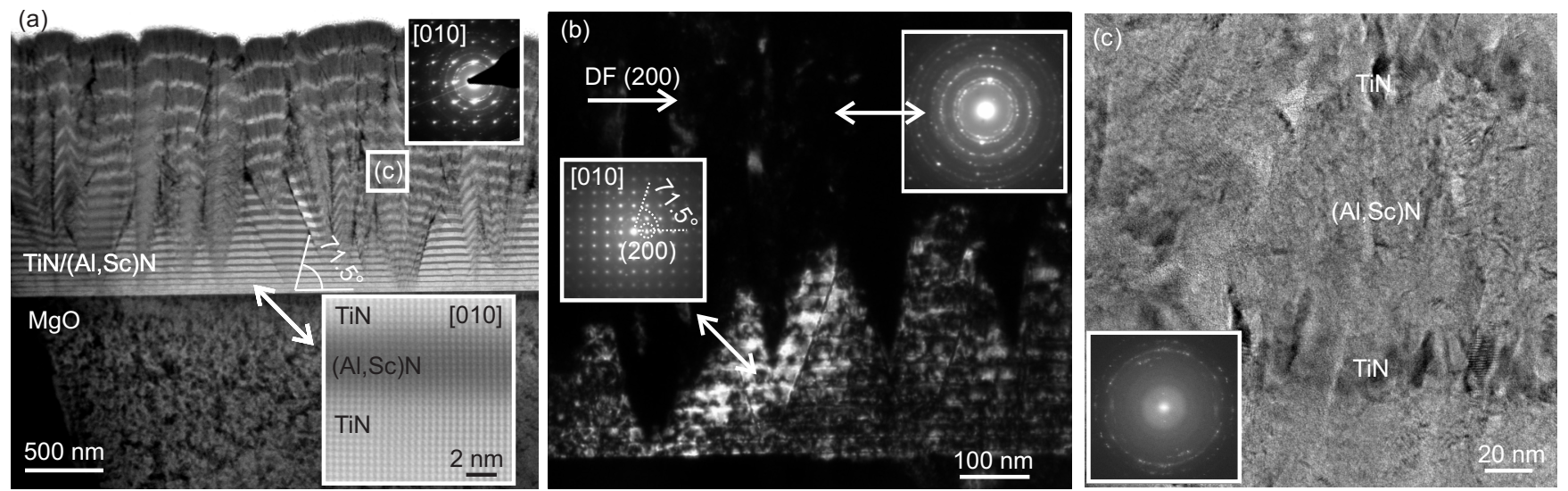

FIG. 1. (a) Overview STEM micrograph showing the entire multilayer stack grown on $\mathrm{MgO}$ (001), with well-defined atomically flat interfaces in the regions of epitaxial rocksalt multilayer growth. (b) DF TEM showing that the boundary between the stabilized cubic multilayer stack and polycrystalline wurtzite grains with random orientations above is governed by a zigzag line, lying mostly on the $\{103\}$ planes. (c) TEM image showing the microstructure of the polycrystalline region marked with rectangle in (a) showing unordered wurtzite (Al,Sc)N grains as confirmed by the inset FFT.

constant of about $4.2 \AA$ is measured for the rocksalt phase. Analyzing the diffraction rings confirms the $(\mathrm{Al}, \mathrm{Sc}) \mathrm{N}$ wurtzite phase to be present there [the measured $d$ spacings of $\sim 3.7 \mathrm{~nm}^{-1}, \sim 4.0 \mathrm{~nm}^{-1}, \sim 4.2 \mathrm{~nm}^{-1}, \sim 5.5 \mathrm{~nm}^{-1}$, and $\sim 6.5 \mathrm{~nm}^{-1}$ correspond well to the low-index wurtzite $(\mathrm{Al}, \mathrm{Sc}) \mathrm{N}$ $\{10 \overline{1} 0\},\{0002\},\{10 \overline{1} 1\},\{10 \overline{1} 2\}$, and $\{11 \overline{2} 0\}$ planes with nominal values of $3.71 \mathrm{~nm}^{-1}, 4.02 \mathrm{~nm}^{-1}, 4.22 \mathrm{~nm}^{-1}, 5.47 \mathrm{~nm}^{-1}$, and $6.43 \mathrm{~nm}^{-1}$, respectively], along with faint traces of randomly oriented cubic grains, stemming from the TiN. Also, the inset selected-area EDP from the region of intact epitaxial growth shows that the V-shaped defects observed in Fig. 1(a) lie on $\{103\}$ planes. Strikingly, the boundary between stabilized epitaxial multilayer growth in the rocksalt structure and the polycrystalline wurtzite regions is mostly defined by those $\{103\}$ planes as well, while flat and parallel-to-growthdirection boundaries are almost entirely absent.

From overview micrographs like in Fig. 1(a), an average value of $\sim 380 \mathrm{~nm}$ for the total thickness of stabilized epitaxial growth was obtained by integrating the area under the zigzag boundary and substrate/film interface for many micrographs covering a sample width of several tens of $\mu \mathrm{m}$. Individually, it can be seen that $(\mathrm{Al}, \mathrm{Sc}) \mathrm{N}$ films with thicknesses of up to $26 \mathrm{~nm}$ and at an average value of $16 \mathrm{~nm}$ were pseudomorphically stabilized in the rocksalt phase successfully. This value is almost 10 times higher than those reported previously on similar systems as AlN/TiN and GaN/TiN, respectively, and was shown to be sensitive to the AlN mole fraction $[13,14]$.

Figure 2(a) shows a BF TEM micrograph of a region with both well-defined cubic multilayer growth and adjacent polycrystalline wurtzite phase. Strong strain contrast is observed within the multilayer stack, mostly in the TiN layers. In order to quantify the strain, geometric phase analysis was employed on atomically resolved micrographs, such as the one in the inset of Fig. 1(a), of each individual layer from bottom to top of the multilayer stack [positions shown in Fig. 2(b)]. As reference, regions of supposedly minimum strain in the lowermost TiN
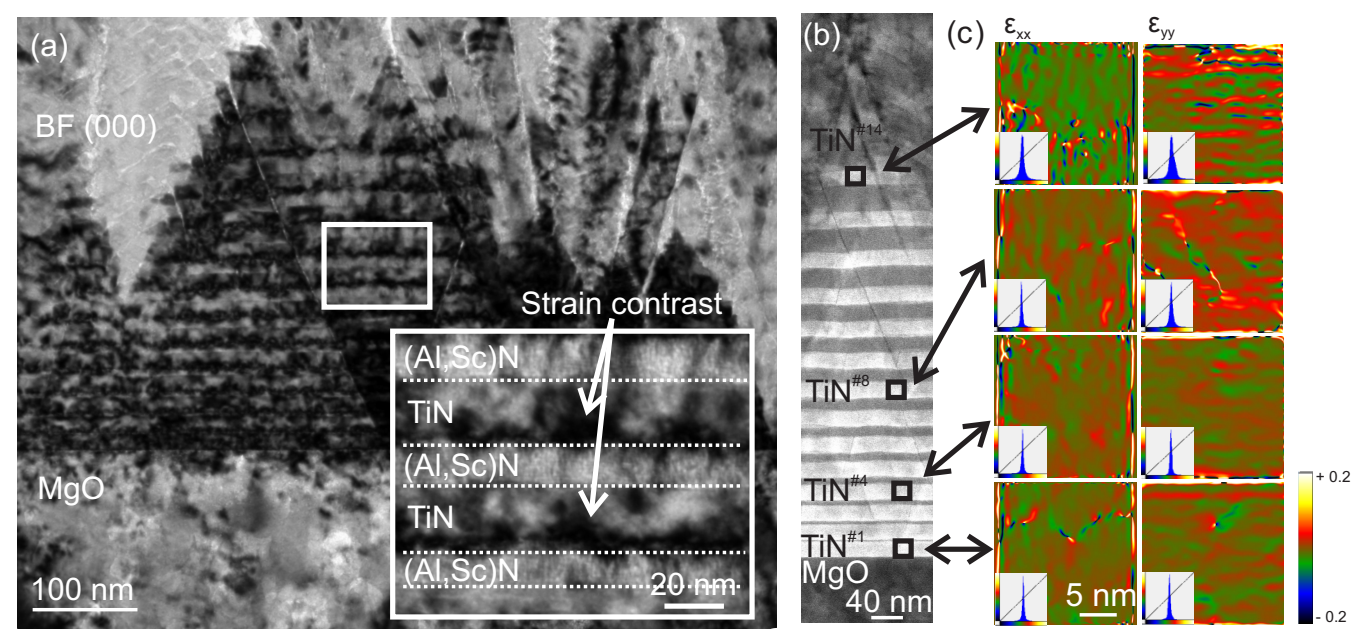

FIG. 2. (a) BF TEM micrograph displaying strain contrast mostly within the TiN layers. Geometric phase analysis of atomically resolved micrographs taken within the TiN layers situated in a vertical line on top of each other along the direction of growth (b) show an increase in lattice strain up to numbers of more than $10 \%$ before the cubic stabilization breaks down (c). 


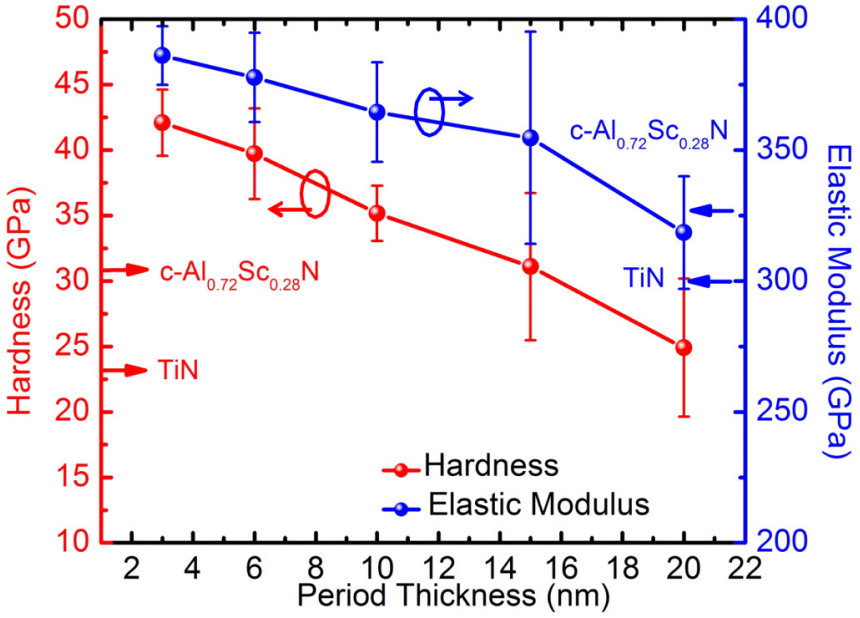

FIG. 3. Hardness, and elastic modulus as measured by nanoindentation and plotted as function of multilayer period thickness and compared to the individual metal and semiconductor compounds in rocksalt phase. The TiN and superlattice films were $300 \mathrm{~nm}$ in thickness each, and the $\mathrm{Al}_{0.72} \mathrm{Sc}_{0.28} \mathrm{~N}$ was a $120 \mathrm{~nm}$ thick stabilized film on $20 \mathrm{~nm}$ TiN/MgO substrate. Hardness values adapted from our previous work [18].

layer were chosen. The results are plotted in Fig. 2(c), showing the $x$ and $y$ components of the strain tensor for comparison from four different TiN layers. As can be seen from the color scale (\% of strain/100) and the inset histograms depicting the distribution of values measured in each map, the strain increases noticeably from base to top of the multilayer stack, with values as high as $\sim 10 \%$ and above in the uppermost TiN layer piece within the region of interest.

This is well in line with metastability theory that suggests that before the phase transition from rocksalt to wurtzite occurs, defects will nucleate, the interfaces will roughen, and coherency-strain energy becomes accumulated within the layers, leading ultimately to failure of stabilization beyond a critical thickness. The strain builds up mostly in the TiN layers, while $(\mathrm{Al}, \mathrm{Sc}) \mathrm{N}$ layers do not show increasing strain with thickness, possibly due its higher elastic modulus as shown in Fig. 3. Also, it is clear that for energetic reasons, the phase transition would occur more readily during the $(\mathrm{Al}, \mathrm{Sc}) \mathrm{N}$ growth itself and not after it is covered with TiN again [13]. That is clearly confirmed by our measurements.

Figure 3 shows hardness and elastic moduli of individual TiN and $\mathrm{Al}_{0.72} \mathrm{Sc}_{0.28} \mathrm{~N}$ films compared to their multilayers with varying individual thicknesses, as measured by nanoindentation. Those values emphasize the potential application of the films as hard protective coating since they compare well with those of ultra-incompressible nitrides which exhibit hardness values of up to $50 \mathrm{GPa}$ and bulk modulus larger than $300 \mathrm{GPa}$ [26]. The increase in both hardness and elastic modulus for multilayers with decreasing period thickness is well in line with the strain measurements, since both coherency-strain hardening [27] and Koehler hardening [28,29] are expected to occur within the multilayers. Koehler hardening describes the decrease in mobility of dislocations in a material with higher elastic modulus, in this case the $\mathrm{Al}_{0.72} \mathrm{Sc}_{0.28} \mathrm{~N}$. Koehler has demonstrated that by using alternate and very thin layers of materials with high and low elastic constants, respectively, very high shearing stresses would be required in order to drive dislocations through the layered stack. According to this, the low-elastic-constant material should be such that perfect dislocations rather than partials occur in bulk specimens of the material, for which several combinations (mostly metals) were suggested [29].

The classical model suggests relaxation of coherency strain by the formation of dislocations, which in the multilayer films should occur just before the phase transition. However, as can be seen from Figs. 1(a) and 2(a), we find V-shaped features crossing through the film and forming the boundary between rocksalt and wurtzite regions, which suggests that they play a role in the relaxation of coherency strain within the layers. In order to analyze their nature, high-resolution STEM in conjunction with EDS mapping was employed. Figure 4(a) shows an overview micrograph of the investigated region. Two defects originating close to (but not exactly at) the substrate film interface can be seen, running diagonally at the already observed angle of about $71.5^{\circ}$ upwards, corresponding to the (103) and (103) lattice planes. Interestingly, it can be seen that those defects have a width beyond a single atomic layer, differing from what would be expected from linear defects as dislocations and stacking faults.

Moreover, further away from the base of the multilayer stack, it can be seen that those defects coincide with a kinking of the layers at their points of crossing the interfaces, and that such kinking appears to have two different "signs" (meaning the parts of the layers separated by the defects appear upward/downward shifted) with respect to a lateral crossing of either the (103) or (103) lattice planes.

Figure 4(b) shows an atomic resolution STEM micrograph of the region marked by a rectangle in Fig. 4(a). It becomes apparent that while a perfect cubic lattice is maintained across the weak-contrast $\{103\}$ planes with a width of $2-4 \mathrm{~nm}$, those regions are depleted of atoms with respect to the neighboring lattice. Indeed, individual elemental EDS maps [(c)-(f)] confirm those regions to constitute voids, while no signs of interdiffusion between the layers can be seen. Further, although we cannot entirely exclude any other form of possible contamination of the substrate surface, the voids do not originate at any sort of dislocation or similar defect at or nearby the substrate-film interface. On the contrary, they seem to appear just during the growth of the first TiN layer on the $\mathrm{MgO}$ [Fig. 4(a)]. A possible source for the formation of the voids could be shadowing during the growth, which has been found before to introduce defects and voids of slightly different nature in comparable nitride superlattices [21,30,31].

Figure 5(a) displays a HRTEM micrograph showing the voids in atomic resolution. It can be seen that these decorate the $\{103\}$ planes in an irregular fashion, with steps of a few atoms in width, and accompanied by edge dislocations of different signs in the surrounding lattice.

The formation of sharp kinks at the crossing between the voids along [103] and the layers becomes most apparent at the interfaces, as can be seen in the lattice-resolved STEM micrograph of Fig. 5(b). The different height of the layers on either side of the voids hints at a slightly different pace of growth. As mentioned above already, Fig. 4(a) shows that this is a regular feature that can be found throughout the multilayer 

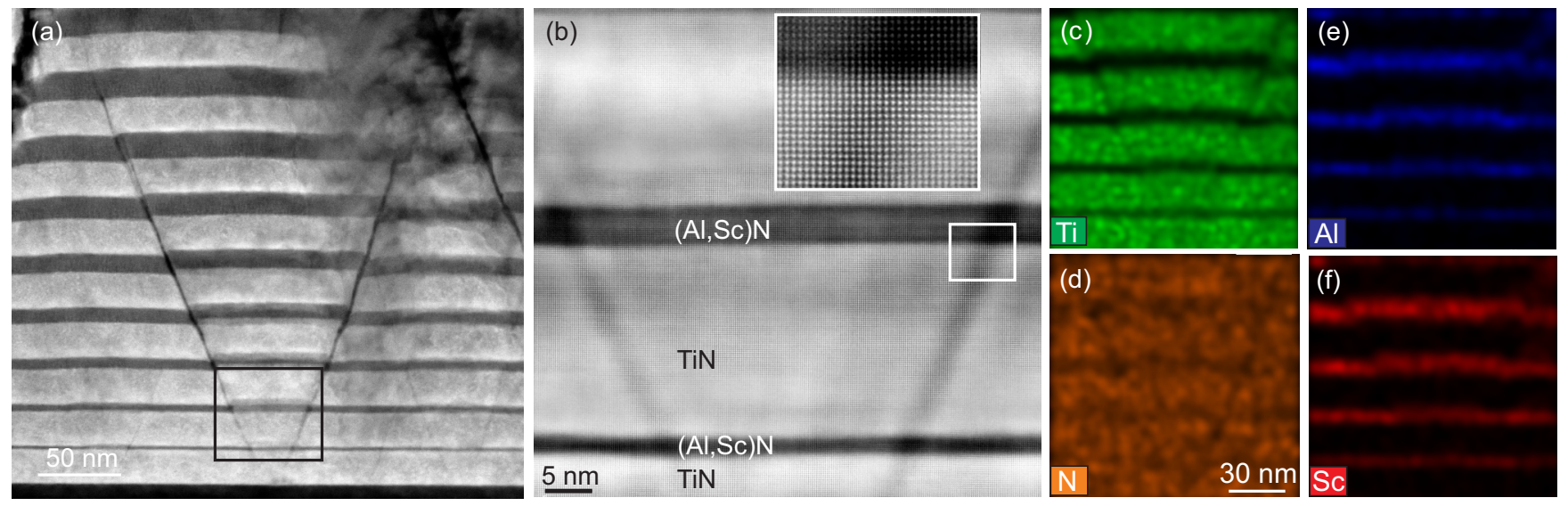

FIG. 4. (a), (b) STEM micrographs of the V-shaped defects on $\{103\}$ planes in different magnifications reveal them to be about $2-4 \mathrm{~nm}$ wide, and devoid of material with respect to the surrounding regions, while still portraying the cubic lattice in both TiN and (Al,Sc)N layers. Individual element EDS maps clearly confirm the void character [(c)-(f)].

growth: Within the " $\mathrm{V}$ " regions, growth is accelerated with respect to the neighboring "inverted-V" regions. We suspect that this is due to an additional growth front perpendicular to the voids, which is substantiated by the facets of the kinks as indicated by the two arrows that lie exactly perpendicular to the [103] orientation of the voids. The additional growth front can at the same time explain the presence of regions devoid of atoms along [103], since those are transported towards that second direction of growth.

A plan-view scanning electron microscopy (SEM) image [Fig. 5(c)] shows tetragonal features corresponding to the pyramidal growth regions bordered by the voids along $\{103\}$ planes. Those appear smooth and sharp with respect to the surrounding areas of random-orientation wurtzite phase.

At the same time, the voids decorating $\{103\}$ planes are observed to form the boundary between epitaxially cubic stabilized multilayer and unordered wurtzite polycrystalline regions beyond [Figs. 1(a) and 2(a)]. Noticeably, almost no flat regions mark that boundary. The B1 (103) plane's projection forms an imperfect (strained) hexagon however, and this might be where some of the first wurtzite grains start to nucleate, appreciating that the wurtzite phase has a larger molar volume than the cubic phase. Indeed, the wurtzite phase's [2 $1 \overline{1} 11]$ projection [corresponding to $(10 \overline{3})$ in cubic notation] yields a very close match to that of the rocksalt [103] zone axis with a mismatch of imperfect hexagonal angle of about $3^{\circ}$ only. The presence of voids further stimulates the random orientation nature of growth beyond that boundary due to the presence of other possible orientations with slightly higher angle mismatch. The more voids accumulate in the film as evidenced by the EDS maps (Fig. 4), the more the phase transition is promoted. Hence, the voids along the $\{103\}$ planes form a trace of the propagating growth and a minimum energy plane between the cubic [103] and hexagonal [21̄111].

Furthermore, the nanoindentation measurements (Fig. 3) show that both the hardness and elastic modulus increase for the entire multilayer stack with respect to the individual TiN and $\mathrm{Al}_{0.72} \mathrm{Sc}_{0.28} \mathrm{~N}$ for decreasing layer thicknesses, which corresponds well with the measured increase in coherency strain for increasing $\mathrm{Al}_{0.72} \mathrm{Sc}_{0.28} \mathrm{~N}$ layer thickness [Fig. 2(c)]. The voids hence allow for the coherency strain to relax just before the phase transformation occurs, instead of the
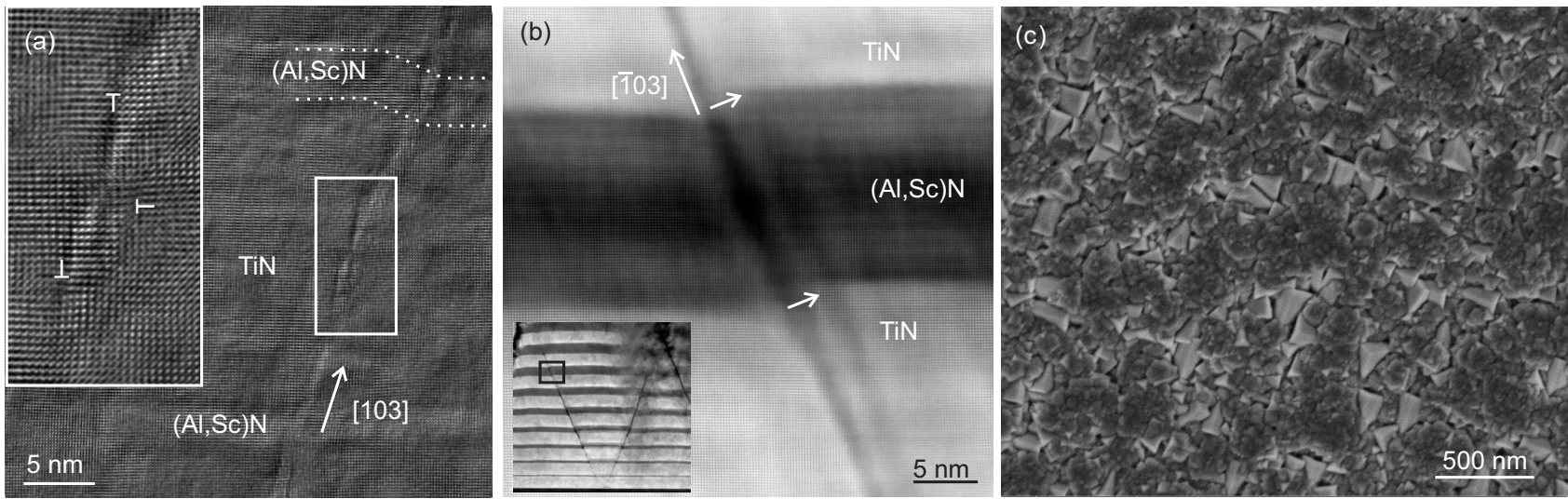

FIG. 5. Analysis of the voids decorating the $\{103\}$ planes. (a) HRTEM micrograph showing irregularly shaped voids decorating the (103) planes with a width of 2-3 nm, accompanied by edge dislocations in the surrounding lattice. (b) Atomically resolved STEM of a region where the voids cross through the TiN/(Al,Sc)N interfaces, separating regions of slightly different growth pace. (c) Plan-view SEM micrograph showing tetragonal features corresponding to the pyramidal growth regions bordered by voids seen in TEM cross section. 
formation of dislocations which is hampered by the Koehler hardening within the $\mathrm{Al}_{0.72} \mathrm{Sc}_{0.28} \mathrm{~N}$ layers.

\section{CONCLUSION}

In summary, the phase transition within pseudomorphic rocksalt-stabilized metastable lattice-matched $(\mathrm{Al}, \mathrm{Sc}) \mathrm{N}$ by epitaxial growth on rocksalt $\mathrm{TiN}$ in thin-film multilayers with systematically increasing $(\mathrm{Al}, \mathrm{Sc}) \mathrm{N}$ interlayer thicknesses has been investigated. Nanoindentation hardness and elastic moduli measurements in line with strain mapping show that coherency-strain hardening and Koehler hardening are mechanisms responsible for the increase of hardness with decreasing multilayer periods. The increase in individual layer thickness is accompanied by the building up of coherency strain, which is released ultimately during the $(\mathrm{Al}, \mathrm{Sc}) \mathrm{N}$ transition to its stable wurtzite phase, and this release is mediated by voids decorating the boundary between rocksalt and wurtzite phase, since the Koehler hardening prevents relaxation by dislocation formation in the $\mathrm{Al}_{0.72} \mathrm{Sc}_{0.28} \mathrm{~N}$ layers due the higher elastic modulus as compared to TiN. This void-decorated boundary was shown to be along $\{103\}$ planes assisting nucleation, and those serve as additional growth fronts and hence separate pyramidal-shaped regions of different growth rates within the rocksalt regions, while at the same time promoting wurtzite growth upon phase transformation since the interface between the cubic [103] and hexagonal [2 $\overline{1} \overline{1} 1$ ] directions forms a minimum energy plane with offset between lattice angles of only about $3^{\circ}$.

\section{ACKNOWLEDGMENTS}

The Knut and Alice Wallenberg (KAW) Foundation is acknowledged for the Electron Microscope Laboratory in Linköping. M.G. and L.H. acknowledge financial support from the Swedish Research Council [RÅC Frame Program (2011-6505), Project Grant No. 2013-4018, and a Linnaeus Grant (LiLi-NFM)] as well as the Swedish Government Strategic Research Area in Materials Science on Functional Materials at Linköping University (Faculty Grant SFO-MatLiU 2009-00971). B.S. and T.D.S. acknowledge financial support from the National Science Foundation and US Department of Energy (Award No. CBET-1048616). M.G. and B.S. acknowledge support from the Swedish Foundation for International Cooperation in Research and Higher Education (STINT). M.G. acknowledges support in specialized TEM sample preparation at the Karlsruhe Nano Micro Facility (Project ID 2015-015-010151) via V. S. K. Chakravadhanula.
[1] S. Strite and H. Morkoc, J. Vac. Sci. Technol. B 10, 1237 (1992).

[2] F. A. Ponce and D. P. Bour, Nature (London) 386, 351 (1997).

[3] S. Uehara, T. Masamoto, A. Onodera, M. Uedo, O. Shimomura, and K. Takemura, J. Phys. Chem. Solids 58, 2093 (1997).

[4] S. H. Tolbert and A. P. Alivisatos, J. Chem. Phys. 102, 11 (1995).

[5] V. L. Solozhenko, O. O. Kurakevych, P. S. Sokolov, and A. N. Baranov, J. Phys. Chem. A 115, 17 (2011).

[6] F. J. Manjon, D. Errandonea, A. H. Romero, N. Garro, J. Serrano, and M. Kuball, Phys. Rev. B 77, 205204 (2008).

[7] S. Adachi, Properties of Semiconductor Alloys: Group-IV, III$V$, and II-VI Semiconductors (John Wiley \& Sons, Chichester, 2009).

[8] W. Kummerle and U. Gradmann, Solid State Commun. 24, 33 (1977).

[9] G. A. Prinz, Phys. Rev. Lett. 54, 1051 (1985).

[10] M. Baleva and E. Mateeva, J. Phys.: Condens. Matter 5, 7959 (1993).

[11] B. Saha, S. Saber, G. V. Naik, A. Boltasseva, E. A. Stach, E. P. Kvam, and T. D. Sands, Phys. Status Solidi B 252, 251 (2015).

[12] B. Saha, S. Saber, E. A. Stach, E. P. Kvam, and T. D. Sands, Appl. Phys. Lett. 109, 172102 (2016).

[13] A. Madan, I. W. Kim, S. C. Cheng, P. Yashar, V. P. Dravid, and S. A. Barnett, Phys. Rev. Lett. 78, 1743 (1997).

[14] V. Rawat, D. N. Zakharov, E. A. Stach, and T. D. Sands, Phys. Rev. B 80, 024114 (2009).

[15] B. Saha, G. V. Naik, S. Saber, C. Akatay, E. A. Stach, V. M. Shalaev, A. Boltasseva, and T. D. Sands, Phys. Rev. B 90, 125420 (2014).

[16] T. D. Sands, Appl. Phys. Lett. 52, 197 (1988).

[17] T. D. Sands, C. J. Palmstrøm, J. P. Harbison, V. G. Keramidas, N. Tabatabaie, T. L. Cheeks, and Y. Silberberg, Mater. Sci. Rep. 5, 98 (1990).
[18] B. Saha, S. K. Lawrence, J. L. Schroeder, J. Birch, D. F. Bahr, and T. D. Sands, Appl. Phys. Lett. 105, 151904 (2014).

[19] J. L. Schroeder, B. Saha, M. Garbrecht, N. Schell, T. D. Sands, and J. Birch, J. Mater. Sci. 50, 3200 (2015).

[20] B. Saha, Y. R. Koh, J. Comparan, S. Sadasivam, J. L. Schroeder, M. Garbrecht, A. Mohammed, J. Birch, T. Fisher, A. Shakouri, and T. D. Sands, Phys. Rev. B. 93, 045311 (2016).

[21] M. Garbrecht, J. L. Schroeder, L. Hultman, J. Birch, B. Saha, and T. D. Sands, J. Mater. Sci. 51, 8250 (2016).

[22] M. Garbrecht, L. Hultman, J. L. Schroeder, B. Saha, and T. D. Sands, Sci. Rep. 7, 46092 (2017).

[23] G. V. Naik, B. Saha, J. Liu, S. M. Saber, E. A. Stach, J. M. K. Irudayaraj, T. D. Sands, V. Shalaev, and A. Boltasseva, Proc. Natl. Acad. Sci. USA 111, 7546 (2014).

[24] M. Garbrecht, L. Hultman, M. H. Fawey, T. D. Sands, and B. Saha (unpublished).

[25] H. Rösner, C. Koch, and G. Wilde, Acta Mater. 58, 162 (2010).

[26] D. Errandonea, Ch. Ferrer-Roca, D. Martínez-Garcia, A. Segura, O. Gomis, A. Muñoz, P. Rodríguez-Hernández, J. LópezSolano, S. Alconchel, and F. Sapiña, Phys. Rev. B 82, 174105 (2010).

[27] A. P. B. Mirkarimi, L. Hultman, and S. A. Barnett, Appl. Phys. Lett. 57, 2654 (1990).

[28] J. S. Koehler, Phys. Rev. 86, 52 (1952).

[29] J. S. Koehler, Phys. Rev. B 2, 547 (1970).

[30] L. Hultman, L. R. Wallenberg, M. Shinn, and S. A. Barnett, J. Vac. Sci. Technol. A 10, 1618 (1992).

[31] L. R. Wallenberg, L. Hultman, M. Shinn, and S. A. Barnett, in Electron Microscopy 92: Proceedings of the 10th European Congress on Electron Microscopy held in Granada, Spain, edited by A. Rios et al. (Secretariado de Publicaciones de la Universidad de Granada, Granada, Spain, 1992), pp. 755-756. 